\title{
Antimicrobial Efficacy of Plant Extracts Combined with Endodontic Sealer on the Growth of Oral Pathogen - An In Vitro Study
}

\author{
Prasada KL and Anusha K* \\ KVG Dental College and Hospital, India
}

*Corresponding author: Anusha K, KVG Dental College \& Hospital, Kurunjibagh, Sullia, India, Email: anukbhat11@gmail.com

\section{Research Article \\ Volume 6 Issue 2}

Received Date: April 26, 2021

Published Date: May 24, 2021

DOI: $10.23880 /$ oajds- 16000294

\section{Abstract}

Aim: The aim of this study is to evaluate the antimicrobial efficacy of synthetic sealing material (Zinc oxide eugenol based sealer- Pulp canal sealer EWT) and medicinal plant extracts (Glycyrrhiza glabra, Zingiber officinale, Cinnamon zeylanicum) individually and in combination against oral pathogen (Enterococcus faecalis).

Methods: The herbal extracts were independently mixed in different ratios with zinc oxide powder, followed by mixing with eugenol to know the effective concentration using antibacterial assay. Different concentrations of each test material were placed into Agar diffusion wells and zones of inhibition were measured against the selected bacteria. The concentration with maximum zone of inhibition for each test material was assessed and grouped as group I- Glycyrrhiza glabra and pulp canal sealer EWT, group II-Zingiber officinale and pulp canal sealer EWT, group III-Cinnamon zeylanicum and pulp canal sealer EWT,Group IV- Plain pulp canal sealer EWT and compared.

Results: It was found that all the groups exhibited varied antimicrobial activity against test microorganism. Highest antimicrobial efficacy was noticed in group III followed by group II, group I and least with group IV samples.

Conclusion: Zinc oxide eugenol based sealer with plant extracts produced inhibitory zones against tested microorganism. Hence the plant extract can be added as one of the component of endodontic sealer, but further clinical trials need to be conducted before recommending them as sealers.

Keywords: Glycyrrhiza glabra; Zingiber officinale; Cinnamon zeylanicum; Zinc oxide eugenol based sealer; Enterococcus faecalis; Agar well diffusion

\section{Introduction}

Microorganisms and their byproducts are the main agents causing endodontic disease [1]. Thus, destruction of these microorganisms in the root canal system is the major goal of endodontic treatment. Effective performance in all phases determines the success of endodontic treatment. Even after thorough chemo-mechanical preparation, micro-organisms still remain in the canal due to the complicated root canal anatomy including accessory canals, isthmus and fines which are inaccessible and the aim of root canal treatment cannot be accomplished completely [2]. Therefore, it is desirous to use sealers with a good sealing ability and antimicrobial activity to eliminate these residual microorganisms [3]. 


\section{Open Access Journal of Dental Sciences}

However, controversies related to antibacterial effects of sealers on common isolated bacteria in infected teeth as well as their degree of cytotoxicity , increased antibiotic resistant strains and side effects caused by synthetic drugs has made researchers to look for herbal alternatives [4,5].

The usage of plants and plant products as medicines is outlined way back from beginning of human civilization. Medicinal plants are rich in Secondary metabolites and essential oils which are of therapeutic importance to humans. Several advantages of using medicinal plants are that they are safe, economical, less toxic, easily available, good shelf life and less chances of developing microbial resistance [6,7].

Cinnamon also known as C. zeylanicum has gained popularity in recent time due its antibacterial activity. Vanillic, caffeic, gallic, protocatechuic, p coumaric, and ferulic acids present in the extracts are the ones which exhibit antibacterial action. It has shown effectiveness against $S$. mutans and E. faecalis organisms [8]. It has also known to show a wide spectrum of action on gram positive and gram negative organism. The $C$. zeylanicum bark is rich in cinnamaldehyde (50.5\%), which has been proven to be active against many pathogenic gram-positive and gram-negative bacteria. Ali, et al. [3] reported cinnamaldehyde as the active agent to inhibit the growth of both antibiotic-sensitive andresistant strains of Helicobacter pylori [3,9].

Ginger belongs to Zingiberaceae family and it has also been used as a medicine from Vedic days due to its antibacterial and antifungal properties. Maekawa, et al. evaluated the antimicrobial efficacy of Glycolic propolis, ginger extract, $\mathrm{Ca}(\mathrm{OH})_{2}, \mathrm{CHX}$ and their combinations against E. faecalis, C. albicans, Escherichia coli and endotoxins in root canals. The ginger extract used in this study, was the dehydrated rhizome of Zingiber officinale, which contains flavonoids, gingerol, shogaol, zingerone, paradol and volatile oil [10].

Liquorice (Glycyrrhiza Glabra) commonly known as Atimaturam is the most commonly used flavouring agent in kampo medicines. The compound glycyrrhizin (or glycyrrhizic acid), found in liquorice, has antiviral, antimicrobial, anti-inflammatory, and anti- carcinogenic. Liquorice extract is efficient in reducing pain and promotes healing in apthous ulcer [11-13].

Though there are various uses of herbs in medicinal field, less studies have been done in the field of dentistry.

Hence, the aim of the study was to evaluate and to compare the antimicrobial efficacy of root canal sealer mixed with herbal extracts against oral pathogen.

\section{Methodology}

The present in vitro microbiological study was conducted in Department of Conservative dentistry and Endodontics, KVG Dental College and Hospital, in collaboration with Department of Microbiology, KVG Medical College and Hospital and KVG Ayurveda Medical College, Sullia. In the present study antimicrobial efficacy of three herbal extracts Glycyrrhiza glabra, Zingiber officinale, Cinnamon zeylanicum in combination with Zinc oxide eugenol based sealer (Pulp canal sealer EWT) was evaluated against Enterococcus faecalis using agar diffusion test.

\section{Tested Herbal extracts}

Three herbal extracts used in this study were methanolic extracts of Glycyrrhiza glabra, Zingiber officinale, Cinnamon zeylanicum.

\section{Procurement of the extract}

The herbal extracts were procured from KVG Ayurveda medical college and Hospital, Sullia. The plant materials (ginger, liquorice, cinnamon) were collected and shade dried. The dried parts of plants were crushed and powdered using electrical grinder. The powdered material (50grams) was extracted with $500 \mathrm{ml}$ methanol.

\section{Test micro-organism: Enterococcus faecalis}

\section{Procedure: Antimicrobial assay}

Powder and liquid ratios were standardized according to the formula given by Tchaou, et al. [14] i. e., $0.2 \mathrm{gm}$ of zinc oxide powder mixed with $0.07 \mathrm{ml}$ of eugenol (pulp canal sealer EWT). Based on this formula herbal powders were measured by weight and mixed in different ratios (Table 1) with zinc oxide powder for effective concentration. Digital weighing machine and a micropipette were used to measure the exact amount of powder and liquid to be dispensed. Each test material thus prepared was mixed with eugenol to obtain a creamy mix and used for antibacterial assay. Different concentrations of each test material were placed into agar diffusion wells and zones of inhibition were measured against all the test microorganisms. The concentration with maximum zone of inhibition for each test material was assessed. The maximum inhibition zones were observed as group I- Glycyrrhiza glabra and pulp canal sealer EWT (60:40), group II- Zingiber officinale and pulp canal sealer EWT (90:10), group III- Cinnamon zeylanicum and pulp canal sealer EWT (70:30), Group IV- Plain pulp canal sealer EWT. 


\begin{tabular}{|c|c|}
\hline Ratio & Weight (in grams) \\
\hline $90: 10: 00$ & $0.18: 0.02$ \\
\hline $80: 20: 00$ & $0.16: 0.04$ \\
\hline $70: 30: 00$ & $0.14: 0.06$ \\
\hline $60: 40: 00$ & $0.12: 0 . .08$ \\
\hline $50: 50: 00$ & $0.1: 0.1$ \\
\hline $40: 60$ & $0.08: 0.12$ \\
\hline Extract & 0.2 \\
\hline Zinc oxide eugenol sealer & 0.2 \\
\hline Zinc oxide & 0.2 \\
\hline Eugenol & - \\
\hline
\end{tabular}

Table1: Different ratios of herbal and Zinc oxide powder. committee for clinical Laboratory Standards was employed in antimicrobial susceptibility testing for the aqueous extract concentrations of each test material. 5Agar media $(100 \mathrm{ml})$ was sterilized in separate conical flasks, cooled and inoculated with $0.1 \mathrm{ml}$ of the respective test bacterial suspension. After thorough mixing, the inoculated medium was transferred into sterilized petri dishes and on solidification of agar medium, four wells of about $6 \mathrm{~mm}$ diameter were punched with a sterilized cork borer. Just before agar diffusion assay each of the test materials with pre-designated concentration was spatulated on a dry pre sterilized glass slab using a cement spatula at room temperature. $200 \mathrm{mg}$ of each test material was back loaded into a sterile $2 \mathrm{ml}$ syringe and placed into the wells. The inoculated bacterial plates were incubated at $37^{\circ} \mathrm{C}$. The diameter of thus obtained inhibition zones were measured after 24 hours using verrnier callipers of accuracy $0.5 \mathrm{~mm}$ (Figure 1).

Agar well diffusion method prescribed by National
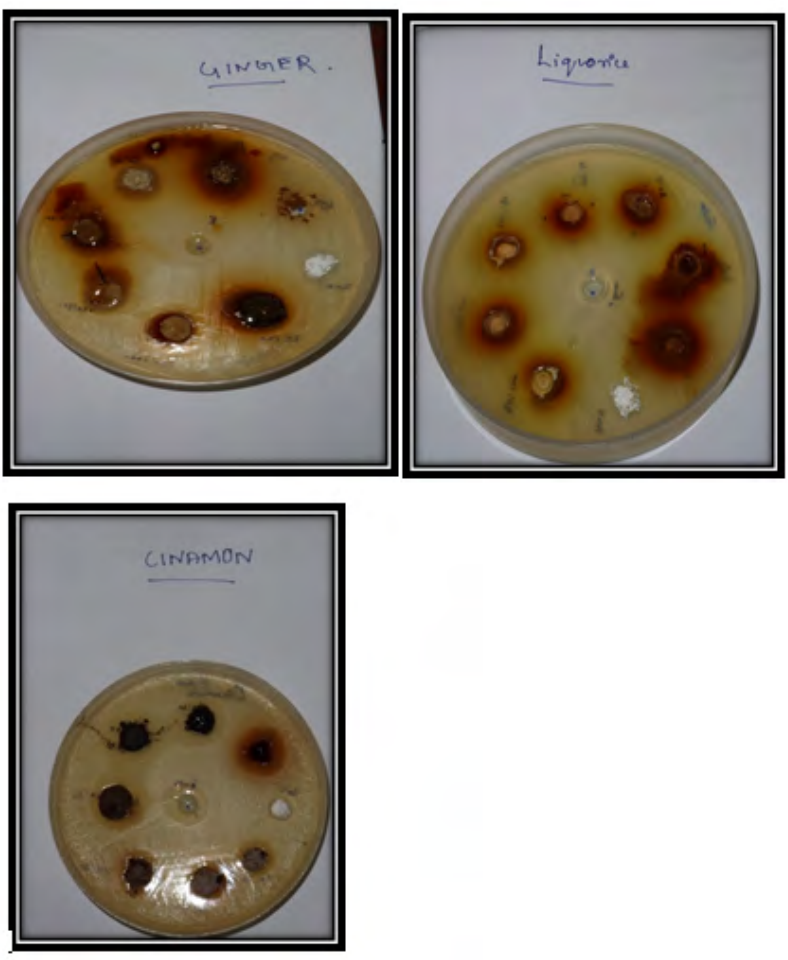

Figure 1:Agar diffusion test showing mean zone of inhibition.

\section{Measuring the Size of Zone of Inhibition}

Growth inhibitoryzones around each tested medicaments were evidenced by lack of bacterial colonization (clearing of agar) adjacent to each group. The most uniform diameter segment of the zone of inhibition was measured, and $6 \mathrm{~mm}$ (diameter of the well) was extracted from the measurement as the cutoff value. All measurements above this value were considered, indicative of significant bacterial growth inhibition. Wider zones of inhibition were interpreted to indicate greater antimicrobial activity of the involved medicaments (Figure 1). 


\section{Statistical Analysis}

Statistical analysis were performed using ANOVA test and POST HOC TUKEY TEST at 0.05 significance level. All data were presented in tabular and bar diagram form. The confidence level of the study was proposed to be $95 \%$; hence, $\mathrm{p}<0.05$ was considered statistically significant.

\section{Results}

Group III- showed maximum zone of inhibition against the tested organism with mean value of 19 at70:30 concentration, followed by group II with mean value of 14.55 at 90:10 concentration, group with mean value 13.8 at 60:40 concentration and group IV with mean value 10 (Table 2, Figure 2).

\begin{tabular}{|c|c|c|c|c|c|}
\hline Study Group & N & Minimum & Maximum & Mean & Std. Deviation \\
\hline Group one & 9 & 0 & 17 & 13.88 & 5.3 \\
\hline Group two & 9 & 0 & 20 & 14.55 & 6.9 \\
\hline Group three & 9 & 0 & 25 & 19 & 7.9 \\
\hline Group four & 9 & 0 & 12 & 10.77 & 0.9 \\
\hline
\end{tabular}

Table 2: Mean Zones of Inhibition of Enterococcus faecalis.

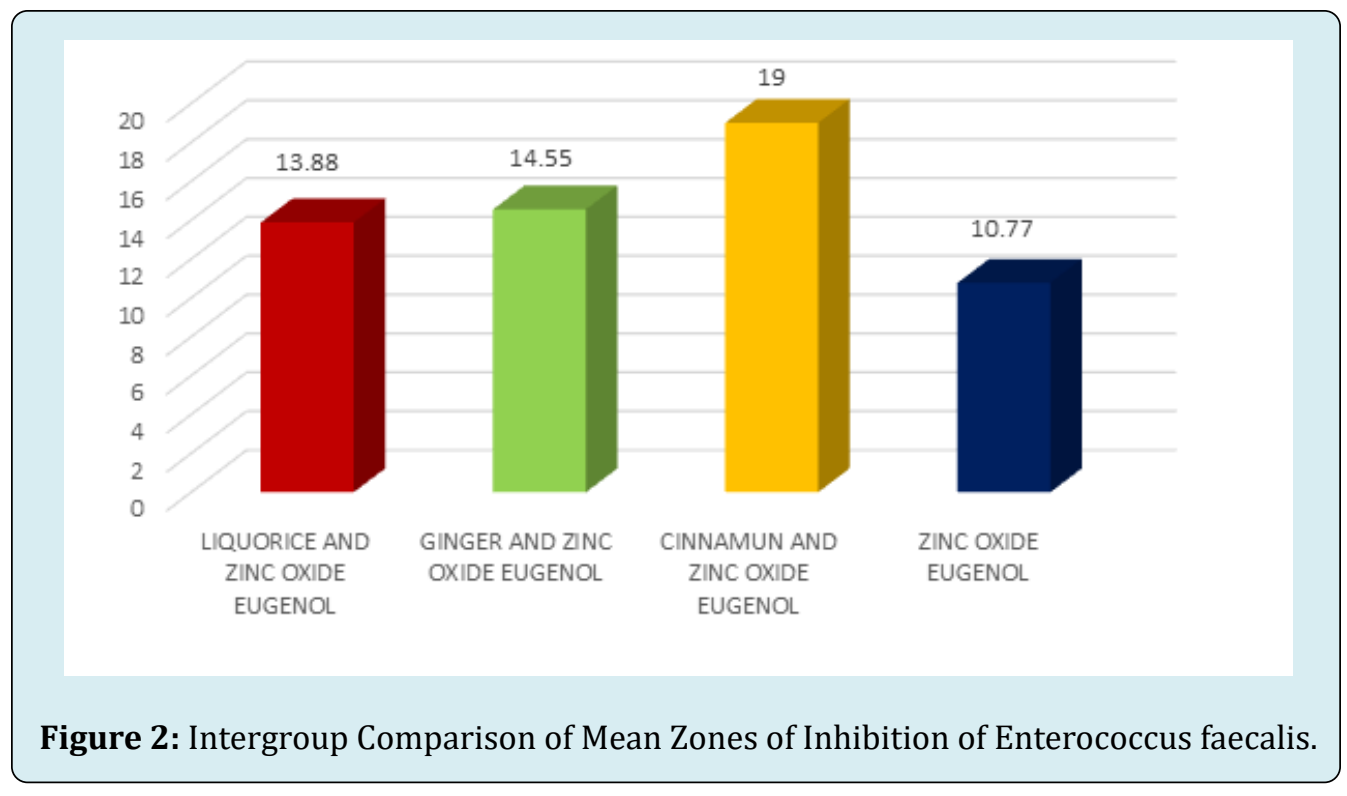

Intergroup comparison of mean zone of inhibition of E. faecalis showed statistically significant results (Table 3 ). Intragroup comparison of mean zone of inhibition of $E$. faecalis showed statistically significant results in group III and group IV (Table 4, Figure 2).

\begin{tabular}{|c|c|c|c|}
\hline Study Group & Mean & Std. Deviation & P value \\
\hline Group one & 13.88 & 5.3 & $0.03^{*}$ \\
\hline Group two & 14.55 & 6.9 & \\
\hline Group three & 19 & 7.9 & \\
\hline Group four & 10.77 & 0.9 & \\
\hline
\end{tabular}

Table 3: Intergroup Comparison of Mean Zones of Inhibition of Enterococcus faecalis. 


\begin{tabular}{|c|c|c|c|}
\hline Study Group & Mean & Std. Deviation & P value \\
\hline Group two & 14.55 & 6.9 & \multirow{2}{*}{0.904} \\
\hline Group one & 13.88 & 5.3 & \\
\hline Group four & 10.77 & 0.9 & \multirow{2}{*}{0.65} \\
\hline Group one & 13.88 & 5.3 & \\
\hline Group three & 19.00 & 7.9 & \multirow{2}{*}{0.24} \\
\hline Group one & 13.88 & 5.3 & \\
\hline Group three & 19.00 & 7.9 & \multirow{2}{*}{0.35} \\
\hline Group two & 14.55 & 6.9 & \\
\hline Group four & 10.77 & 0.9 & \multirow{2}{*}{0.49} \\
\hline Group two & 14.55 & 6.9 & \\
\hline Group four & 19.00 & 7.9 & \multirow{2}{*}{$0.02^{*}$} \\
\hline Group three & 10.77 & 0.9 & \\
\hline
\end{tabular}

Statistical analysis: Post HOC Tukey test. * Statistically significant if $\mathrm{P}<0.05$.

Table 4: Intragroup Comparison of Mean Zones of Inhibition of Enterococcus faecalis.

\section{Discussion}

Elimination of bacteria by cleaning, shaping, and obturation of the root canal system is the objective of root canal treatment. So the root canal sealers with good sealing ability and antimicrobial activity with low toxic effects, which are desirous to kill residual microorganisms inside the canal are recommended [5]. Hypersensitivity, immune suppression, and allergic reactions are some of the adverse effects associated with antimicrobial agents, and the continuous evolution of bacterial resistance has necessitated the researchers to search for medicinal plant alternatives as antimicrobial agents [8].

Medicinal plants are rich sources of bioactive compounds such as alkaloids, flavonoids, and phenolic compounds. Owing to their ability to combat new developing drug resistant pathogens, for herbal preparations it is always a welcome in present day science [4]. There are insufficient scientific reports that indicate the antimicrobial activities of herbal extracts mixed with endodontic root canal sealers. Hence, the aim of the study was to evaluate and compare the antimicrobial efficacy of endodontic sealer mixed with herbal extracts.

The most commonly used root canal sealers in endodontics are zinc-oxide-eugenol-based sealer. As it has antimicrobial activity against $E$. faecalis, $S$. mutans, E. coli and S. aureus. Hashieh, et al. (1999) studied the useful effects of eugenol and stated that the amount of eugenol released in the periapical area immediately after placement was 104 and falls to $10-6$ after $24 \mathrm{hrs}$, reaching zero after one month. Within these concentrations it is said to have anti-inflammatory and analgesic properties. Considering these valid points, the present study was designed to evaluate whether there is any improvement in the antibacterial activity by combining the herbal extracts with ZOE [5]. So in this study pulp canal sealer EWT (zinc oxide eugenol based sealer) composed of Powder: Zinc oxide, silver, Liquid: Eugenol, Balsam, Canada was taken.

Methanolic extracts were shown to have a greater activity compared to ethanolic or aqueous extracts because more phytoconstituents are leached out from it when compared to ethanolic extracts. Hence, methanolic extracts of three herbal plants were prepared as test medicaments $[4,15]$.

Enterococcus faecalis (a gram-positive coccus) was chosen as the test organism because it is a facultative anaerobe that is the most resistant species in the oral cavity and the possible cause of failure of root canal treatment. Sundqvist, et al. has quoted that $38 \%$ of failed root canal systems were contaminated with $E$. faecalis. Therefore, it might be advantageous if the sealer exerts some antimicrobial activity as the last element in treatment regimen $[2,10,13]$.

Antimicrobial efficacy of any natural or synthetic agent can be evaluated by Broth dilution method, Agar dilution method, Disc diffusion method, Cup plate method or Agar well diffusion method and Ditch plate method.8However, agar well diffusion method was used in the present study as it depends upon the diffusion of the tested material to such an extent that growth of the added microorganism is prevented entirely in a zone around the well containing the test material [5]. 


\section{Open Access Journal of Dental Sciences}

The result of the present study showed that zinc oxide eugenol based root canal sealer (pulp canal sealer EWT) combined with Cinnamon showed highest antimicrobial efficacy in descending order followed by ginger and sealer combination and liquorice and sealer combination.

The high antimicrobial efficacy of cinnamon combination in group III may be attributed due to the presence of vanillic, caffeic, gallic, protocatechuic, p coumaric, and ferulic acids in the extract. It has shown effectiveness against $S$. mutans and E. faecalis organisms [6,8]. The bark is rich in cinnamaldehyde $(50.5 \%)$, which has been proven to be active against many pathogenic gram-positive and gramnegative bacteria. Compared to plain ZOE, more antibacterial activity was noticed when ZOE is mixed with herbal extracts. This results were in accordance with study by Cinthura C, et al. [10] Gupta Wadhwa, et al. [16] Sabarathinam, et al. [6] Veerale Panchal et al. [8], Gupta, et al. [17] have done done studies on with cinnamon zylanicum as irrigant, intracanal medicant and have obtained the similar result.

Group II sample exhibited better antibacterial activity compared to group I and group IV , this may be due to presence of, the pungent substances namely gingerol, shogaol, zingerone, paradol and volatile oilin it, studies tell that The most effective antimicrobial constituent was found to be citral inhibiting growth of both gram-negative and gram-positive bacteria [15].

Group I sample showed better result than group group IV due to the presence of liquorice. Glycyrrhizin, a diglucuronide, is the active principle showing better antibacterial effect as compared to ZOE sealer alone [12].

Zinc oxide (Pulp canal sealer-powder) component was resistant and eugenol (pulp canal sealer-liquid) exhibited antibacterial activity in this study. Zinc oxide Eugenol based sealer showed least inhibition zone.Amongst the plain extract which was tested for antibacterial sensitivity Cinnamon showed maximum inhibition zone followed by ginger and liquorice.

The antimicrobial efficacy of any test agent depends upon its extent of diffusion capacity through the solid agar medium which will show the inhibitory zones. The variability in the inhibition zones noticed in the present study can be attributed to the formulations used that provided a compatible mix with zinc oxide eugenol. Studies have also been done with ethanolic or methanolic extract forms of herbs and has shown that these alterations can either increase or decrease the actual antimicrobial activity of these formulations. Another reason could be the type of organism selected. In the present study, lyophilized forms were used whereas in the earlier studies the organisms have been isolated directly from the root canals [18].

\section{Conclusion}

On the basis of the results, observations, and statistical analysis, the following conclusions could be drawn: Zincoxide-eugenol-based root canal sealer (pulp canal sealer EWT) when mixed with the three tested herbal extracts, showed maximum zone of inhibition for Cinnamon zylanicum followed by, Zingiber officinale and then Glycyrrhiza glabra. However the synergic effect needs to be further evaluated for clinical application.

\section{References}

1. Al Khatib ZZ, Baum RH, Morse DR, Yesilsoy C, Bhambhani $S$, et al. (1990) The antimicrobial effect of various endodontic sealers. Oral Surgery, Oral Medicine, Oral Pathology 70(6): 784-790.

2. Beshr KA, Abdelrahim RA (2019) Antibacterial efficacy of Allium sativum (garlic) and chitosan incorporated into two root canal sealers against Enterococcus faecalis: comparative study. Tanta Dental Journal 16(2): 94.

3. Ali WM, Tawfiq NOM, Sulieman RT (2012) Evaluation of antibacterial effects of ginger extract when used as one component of the root canal sealers; (An in vitro study). Tikret Journal of Pharmaceutical Sciences 8(2).

4. Devi MT, Saha S, Tripathi AM, Dhinsa K, Kalra SK (2019) Evaluation of the Antimicrobial Efficacy of Herbal Extracts Added to Root Canal Sealers of Different Bases: An In Vitro Study. Int J Clin Pediatr Dent 12(5): 398-404.

5. Navaneet S, Muktineni S, Avula SSJ, Kakarla P, Kommineni HC, et al. (2019) Antimicrobial efficacy of herbal extracts combined with zinc oxide eugenol as an obturating material in primary teeth-An in vitro study. Journal of Dr. NTR University of Health Sciences 8(4): 244-249.

6. Sabarathinam J, Muralidharan NP, Pradeep (2018) Antimicrobial efficacy of four different intracanal medicaments on contaminated extracted teeth: In vitro study. Drug Invention Today 10(2): 3026-3029.

7. Pujar M, Patil C, Kadam A (2011) Comparison of antimicrobial efficacy of Triphala,(GTP) Green tea polyphenols and $3 \%$ of sodium hypochlorite on Enterococcus faecalis biofilms formed on tooth substrate: in vitro. Journal of International Oral Healt 3(2): 23-29.

8. Panchal V, Gurunathan D, Muralidharan NP (2020) Comparison of antibacterial efficacy of cinnamon extract, Neem extract as irrigant and sodium hypochlorite against Enterococcus fecalis: An in vitro study. Indian 
Journal of Dental Research 31(1): 124-128.

9. Vinothkumar TS, Rubin MI, Balaji L, Kandaswamy D (2013) In vitro evaluation of five different herbal extracts as an antimicrobial endodontic irrigant using real time quantitative polymerase chain reaction. J Conserv Dent 16(2): 167-170.

10. Cinthura C, Geetha RV (2018) Evaluation of antimicrobial activity of endodontic sealers in combination with cinnamon oil. Drug Invention Today 10(2): 2933-2936.

11. Gupta VK, Fatima A, Faridi U, Negi AS, Shanker K, et al. (2008) Antimicrobial potential of Glycyrrhiza glabra roots. J Ethnopharmacol 116(2): 377-380.

12. Kaomongkolgit R, Jamdee $\mathrm{K}$, Pumklin J, Pavasant $\mathrm{P}$ (2013) Laboratory evaluation of the antibacterial and cytotoxic effect of alpha-mangostin when used as a root canal irrigant. Indian Journal of Dentistry 4(1): 12-17.

13. Bipin C, Chitra PB (2013) In vitro antibacterial activity of seven spices against clinical isolates of Enterococci.

14. Tchaou WS, Turng BF, Minah GE, Coll JA (1996) Inhibition of pure cultures of oral bacteria by root canal filling materials. Pediatr Dent 18(7): 444-449.

15. Mistry KS, Sanghvi Z, Parmar G, Shah S (2014) The antimicrobial activity of Azadirachta indica, Mimusops elengi, Tinospora cardifolia, Ocimum sanctum and $2 \%$ chlorhexidine gluconate on common endodontic pathogens: An in vitro study. Eur J Dent 8(2): 172-177.

16. Wadhwa GA, Wadhwa J, Duhan J (2016) Comparative evaluation of antimicrobial efficacy of three herbal irrigants in reducing intracanal E. faecalis populations: An in vitro study. J Clin Exp Dent 8(3): 230-235.

17. Gupta A, Duhan J, Tewari S, Sangwan P, Yadav A, et al. (2013) Comparative evaluation of antimicrobial efficacy of Syzygium aromaticum, Ocimum sanctum and Cinnamomum zeylanicum plant extracts against E nterococcus faecalis: a preliminary study. Int Endod J 46(8): 775-783.

18. Mandal S, DebMandal M, Saha K, Pal NK (2011) In vitro antibacterial activity of three Indian spices against methicillin-resistant Staphylococcus aureus. Oman Med J 26(5): 319-323. 